\title{
Understanding and modulating the competitive surface-adsorption of proteins through coarse-grained molecular dynamics simulations
}

\author{
Pol Vilaseca, ${ }^{a}$ Kenneth A. Dawson, ${ }^{b}$ and Giancarlo Franzese* ${ }^{*}$
}

It is now well accepted that cellular responses to materials in a biological medium reflect greatly the adsorbed biomolecular layer, rather than the material itself. Here, we study by molecular dynamic simulations the competitive protein adsorption on a surface (Vroman effect), i.e. the non-monotonic behavior of the amount of protein adsorbed on a surface in contact with plasma as a function of contact time and plasma concentration. We find a complex behavior, with regimes during which small and large proteins are not necessarily competing between them, but are both competing with others in solution. We show how the effect can be understood, controlled and inverted.

\section{Introduction}

When nanoparticles are in contact with blood plasma, or other biological fluids, biomolecules rapidly coat the bare surface in a relatively selective manner $\underline{\underline{1}}$. It is increasingly accepted that the early biological responses to nanoparticles will be determined by the adsorbed biomolecules rather than the pristine surface alone ${ }^{2} \underline{\underline{-4}}$. Because of their size $\frac{2,5}{2}$ nanoparticles are trafficked by active transport processes throughout the organism, using the information from the protein sequences associated with the surface of nanoparticles. Unlike the situation for flat macroscopic surfaces say of medical implants, for nanoparticles the protein environment changes in different compartments of cells and organs, as the nanoparticle traffics. This has lent urgency to the modern interest in understanding the phenomenon at a more fundamental level ${ }^{2}$. Still, we can learn a lot from an understanding of the process for flat surfaces ${ }^{-}$. Studying the adsorption of fibrinogen on a surface in contact with blood plasma, Vroman found that the surface concentration of fibrinogen displays a maximum at an intermediate contact time, indicating that fibrinogen is replaced with time by one or more families of different proteins ${ }^{7}$. The phenomenon is not specific to fibrinogen, but is a general effect for many other proteins ${ }^{8,9}$. The plasma proteins compete for the occupation of the surface, resulting in a sequential competitive adsorption, known as the Vroman effect.

The effects depends on numerous factors such as the plasma dilution, the $\mathrm{pH}$, the temperature, the surface charge and the specific surface chemistry $\underline{10}$. In highly concentrated plasma, the sequential adsorption takes place in seconds, but it takes several minutes when the plasma is diluted ${ }^{11}$. The effect has been documented both on hydrophilic and hydrophobic inter-

a Departament de Física Fonamental, Facultat de Física, Universitat de Barcelona, Diagonal 645, 08028, Barcelona, Spain. E-mail: gfranzese@ub.edu

${ }^{b}$ Center for BioNano Interactions (CBNI), University College of Dublin, Ireland.E-mail: Kenneth.A.Dawson@cbni.ucd.ie faces 11,12 being more evident the more hydrophilic the material, but with stronger protein binding the more hydrophobic the surfaces 13,14 . However, no universality is found and the results strongly depend on the details of the experiments ${ }^{15}-17$. It is generally accepted that proteins with smaller molecular weight and at higher concentration adsorb first to the surface, but later are replaced by other proteins with, generally, larger molecular weight and size. After the adsorption, the protein can undergo conformational changes and denaturation, especially at a hydrophobic interface, eventually leading to irreversible adsorption 12 .

Many experimental techniques have been used to investigate the effect from blood plasma or model solutions with a limited number of components and many models have been proposed to rationalize the experiments $11,12,18-21$. However, the mechanisms of the phenomenon are still debated and no existing model can fully explain it $17,22,23$.

Volumetric effects, due to non-deformable proteins trying to fit on the available surface, can account for competitive adsorption of proteins 17 . However, they do not reproduce the maxima of absorption of the Vroman effect. This maxima is, instead, rationalized by models based on kinetic equations. Some of these models include coupled mass transport equations ${ }^{18}$. In all of them, to each kind of protein in solutions, there are associated different adsorptions/desorption rate constants. These processes are modeled as reversible by some authors 24 . Others, to fit better the experiments, assume that the adsorption can become irreversible with a "reaction" rate constant $^{18}$. Due to the difficulty for this approach to describe the variety of experimental results, some models include also a "displacement" rate constant of a reversibly adsorbed protein by a protein with a higher surface affinity $20,21,25$. However, these models are unable, in general, to describe solutions at low concentration, where the surface coverage is controlled by diffusion 21 , and cannot rationalize the different desorption behavior observed for sorbent-free with respect to sorbent- 
bearing washing solutions 11 .

The latter observation inspired LeDuc et al. to include also a "liberation" rate constant of semipermanently adsorbed protein by contact with a bulk protein 11 . To simplify the model, the authors made strong approximations, likely to be incorrect, assuming that adsorbed proteins do not diffuse on the surface and that the displacement and liberation rate constant do not depend on the incoming protein 11 . They applied the model to rationalize data of a ternary solution mixture with albumin, high molecular weight kininogen and fibrinogen, accounting also for the deformation of the semipermanently adsorbed proteins. As a result, LeDuc et al. found that, to fit the data, the first two proteins should occupy approximately fourfold more space in the semipermanent state while fibrinogen would have a much smaller change.

This is at variance with what recent experiments show for rod-like proteins as the fibrinogen. This elongated protein, although deforms less then albumin when adsorbed on an extended surface, can undergo a large rearrangement from an initial "lying down" stage (with its long axis parallel to the surface) to a "standing up" conformation (long axis perpendicular to the surface). This conformational change results in a large difference in the occupied surface 26 .

While the models based on kinetic equations are useful to qualitatively reproduce the experimental data by fitting the rate constants, they are less instructive about the mechanisms that at molecular level control the phenomenon. To give an insight into how the competition between sizes, bulk concentrations, surface affinities, diffusion constants and conformational changes combines to give rise to the Vroman effect, we devise here a coarse-grained model of a ternary protein solution mixture in contact with a hydrophobic surface.

\section{The Model}

A full atom simulation of competitive adsorption of proteins from a multicomponent mixture is at present time unfeasible for several reasons. Each protein is made of a large number of amino acids (e.g., 585 for human serum albumin and more than 2800 for the human fibrinogen) and is hydrated by thousands of water molecules. As a consequence, a fully atomistic Molecular Dynamics (MD) simulations of one single protein adsorption on a surface with explicit water is limited to a few hundreds of $\mathrm{ns}^{27}$. This time scale is at least five orders of magnitude smaller than the one necessary to observe competitive adsorption. Moreover, the simulations should be for thousands of proteins.

This challenging task can be undertaken by coarse-graining the system. Coarse-graining can be performed at different levels $\frac{28}{}$. However, modeling a full layer of adsorbed proteins on an extended surface urges to reduce drastically the degrees of freedom. A common strategy is to consider implicit water and to represent the protein as a single particle. As we will explain in the following, this approach does not prevent us from taking into account the possibility of conformational changes. We now describe the details of the model with the approximations we make to reduce the complexity of the problem, bearing in mind that our aim is to show that the competitive adsorption can be understood in terms of a general mechanism, regardless the specific details of the real interactions in the system.

We consider the three most abundant proteins in human blood: albumin, immunoglobulin- $\gamma$ and fibrinogen, for which competitive adsorption on hydrophobic surfaces has been observed ${ }^{16,29}$. The model assumes an implicit solvent and includes through effective potentials the specific energetic and entropic effects of the water hydrating the proteins and the surface ${ }^{30}$, as well as those effects due to the charge distribution on the protein surface or the counter ions in the solution 31 . This method has been validated in many specific cases (e.g., see ${ }^{32.33}$ ) and follows a general approach that has led to the well established DLVO theory (e.g., see ${ }^{34-36}$ ).

Albumin (Alb) is a globular protein, with an almost spherical shape. The immunoglobulin- $\gamma(\operatorname{IgG})$ has a structure that resembles the shape of a Greek letter " $\gamma$ " and can be roughly approximated with a sphere. We model protein-protein interaction for the two spherical proteins as

$$
V_{i, j}(r) \equiv \varepsilon_{i, j}\left(\frac{\sigma_{i, j}}{r}\right)^{24}
$$

where $r$ is the protein-protein distance, $\varepsilon_{i, j}$ the characteristic interaction energy between protein $i$ and protein $j$, where each index can be $A$ for Alb or $I$ for $\operatorname{IgG}$, and $\sigma_{i, j} \equiv R_{i}+R_{j}$, with $R_{i}$ radius of protein $i$. Attraction among proteins is not included at this level of description, as it is small compared to proteinsurface interaction and the protein solution is stable 18 .

The fibrinogen (Fib) in its folded conformation is rod-like. We approximate it with an elongated ellipsoid, with two principal axes of rotation, that can assume two different conformations, one "lying down" and another "standing up" on the surface (Fig. 1). This idea is consistent with experiments 26 and has been used in Monte Carlo simulations with potentials within the DLVO theory $\underline{36}$. Here the two different conformations are encoded in an effective way through soft-core Fib"protein $i$ " potentials,

$$
V_{F, i}(r) \equiv \varepsilon_{F, i}\left[\left(\frac{\sigma_{F, i}}{r}\right)^{24}+\frac{3}{1+\exp \left(30\left(r-\delta_{F, i}\right) / \sigma_{A}\right)}\right]
$$

where $i=A, I, F$ stands for Alb, IgG and Fib, with $\sigma_{F, i} \equiv$ $R_{F}+R_{i}$ corresponding to the interaction along the short axis, $\delta_{F, i} \equiv \delta_{F}+\delta_{i}$ corresponding to the interaction along the long axis, $\delta_{A} \equiv R_{A}, \delta_{I} \equiv R_{I}, \delta_{F}$ the long axis of Fib, and $\varepsilon_{F, i}$ the characteristic interaction energy of Fib with the protein $i$. The protein-protein interaction with the Fib along the short axis is chosen energetically unfavorable with respect to that along the 
(a)

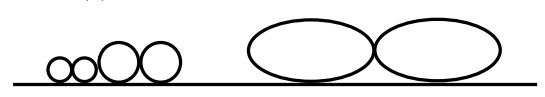

(c)

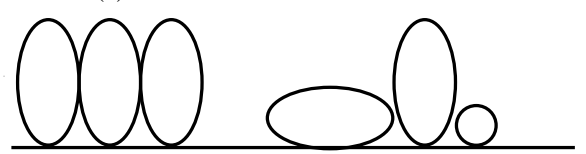

Fig. 1 Schematic representations of different proteins adsorbed on the surface. (a) Alb (smaller) and IgG (larger) are approximated as globular proteins with radiuses $R_{A}<R_{I}$. In all the panels the continuous line represents the surface profile. (b) Fib is represented as an ellipsoid with a short axis $R_{F}$ and a long axis $\delta_{F}$. Fib can assume different conformations: "lying down", as in (b), or "standing up", as in (c), possibly giving rise to mixed configurations, as in (d).

long axis, because the latter offers more binding points to the surface.

Alb has an isoelectric point (IEP) at approximately $\mathrm{pH}$ $5.0^{37}$. By considering a $\mathrm{pH} 5.0$, we minimize the charge interaction for Alb. Hence, the interaction of Alb with the surface is modeled with a short range attraction, that can be thought as mainly due to the entropic gain for water exclusion at the interface,

$$
V_{A, S}(z) \equiv 4 \varepsilon_{A, S}\left(\left(\frac{\sigma_{A}}{z}\right)^{24}-\left(\frac{\sigma_{A}}{z}\right)^{12}\right)
$$

where $z$ is the distance between the center of mass of the protein and the surface and $\varepsilon_{A, S}$ is the attractive energy between Alb and the surface, related to the binding affinity and the dissociation constant. Here $\sigma_{A} \equiv R_{A} / 2^{1 / 12}$, with $R_{A}$ radius of the Alb, takes into account that Alb is a globular protein whose conformation may become distorted on interaction with the surface, resulting in an effective way to take into account conformational changes, as discussed, e.g., in Ref. ${ }^{26}$. The choice of the $24-12$ potential is a simple way of encoding the short range attraction of the Alb-surface interactions. Despite this rough approximation, our results support a posteriori this assumption, consistent with the general idea of Statistical Physics that the details of an attractive interactions do not affect the qualitative results as long as the attractive energy and range of the interaction are preserved ${ }^{38}$.

Since both (monoclonal) $\operatorname{IgG}^{39}$ and Fib $^{37}$ have an IEP at approximately pH 5.5, at the chosen $\mathrm{pH} 5.0$ they are charged. Following other authors, e.g., Ref. $\stackrel{40}{ }$, we consider that the charged proteins, IgG and Fib, have an effective interaction with the surface modelled by a Lennard-Jones potential

$$
V_{i, S}(z) \equiv 4 \varepsilon_{i, S}\left(\left(\frac{\sigma_{i}}{z}\right)^{12}-\left(\frac{\sigma_{i}}{z}\right)^{6}\right)
$$

where $\varepsilon_{i, S}$ is the attractive energy between the protein $i=I, F$ and the surface, and $\sigma_{i} \equiv R_{i} / 2^{1 / 6}$ accounts for the possible distortion of the protein in contact with the surface as a consequence of protein conformational changes due to the adsorption 26.

When adsorbed, Alb, IgG and Fib in its "standing up" conformation occupy a surface $2 \pi R_{i}^{2}$, with $i=A, I, F$, respectively. The Fib in its "lying down" conformation occupies a surface $2 \pi \delta_{F}^{2}$.

To account for the different diffusive behaviors of different proteins in absence of external flow, we calculate the hydrodynamic radius $R_{i}^{H}$ of each protein $i$, under the assumption that the proteins can be approximated by a sphere, through the Einstein-Stokes equation $D_{i}=\frac{k_{B} T}{6 \pi \eta R_{i}^{H}}$, where $D_{i}$ is the experimental diffusion coefficient, $\eta$ the viscosity of the medium, $k_{B}$ the Boltzmann constant, $T$ the absolute temperature. Next, we identify $R_{A}=R_{A}^{H}, R_{I}=R_{I}^{H}$, and $\delta_{F}=R_{F}^{H}$, while $R_{F}$ is set by imposing that the experimental surface concentration found for Fib corresponds to its close packing configuration in the "standing up" conformation 18 . These conditions give $R_{A}=3.55 \mathrm{~nm}, R_{I}=5.51 \mathrm{~nm}, R_{F}=9.29 \mathrm{~nm}$ and $\delta_{F}=11 \mathrm{~nm}$. Protein masses $M_{A}=67 \mathrm{kDa}, M_{I}=150 \mathrm{kDa}, M_{F}=340 \mathrm{kDa}$, necessary to determine the time scales, are known from experiments 13 .

Because we include only repulsive protein-protein interaction, for sake of simplicity we set all the protein-protein $\varepsilon_{i, j}=\varepsilon_{A, S}$. Protein-surface attraction energy $\varepsilon_{i, S}$ can be calculated from the adsorption rate constants 18 . These rates are proportional to the probability for a protein $i$ to attach to the nearby surface

$$
P_{i} \propto \exp \left(\frac{\varepsilon_{i, S}}{k_{B} T}\right) .
$$

However, the $\varepsilon_{i, S}$ in physical units are not known a priori. Hence, we consider the relative probabilities for different proteins $\frac{P_{i}}{P_{j}} \propto \exp \left(\frac{\varepsilon_{i, S}-\varepsilon_{j, S}}{k_{B} T}\right)$, from which is possible to determine the values of the different energies as

$$
\frac{\varepsilon_{j, S}}{\varepsilon_{A, S}}=1-\frac{k_{B} T}{\varepsilon_{A, S}} \ln \left(\frac{P_{A}}{P_{j}}\right)
$$

adopting $\varepsilon_{A, S}$ for Alb as the energy units. We set $\varepsilon_{A, S}$, the only free parameter of our model, by comparing our simulations results with experiments at ambient temperature, and get $\varepsilon_{I, S}=2.79 \varepsilon_{A, S}$ and $\varepsilon_{F, S}=6.08 \varepsilon_{A, S}$ by adopting the adsorption rate constants as in the theoretical model of Lu et al. 18 , that reflect the experimental observation that Fib has the strongest affinity for several surfaces and albumin the weakest. 


\section{The Numerical Method}

We perform MD simulations at constant $T$, constant volume $V$ and constant number of proteins $N_{i}$, in a parallelepiped with two square faces and four rectangular faces. A square face is occupied by the attractive surface, the other by a wall interacting with the proteins through the repulsive part of the potential in Eq. (3). We apply periodic boundary conditions (pbc) along the four rectangular faces. The volume concentrations of proteins is taken to match the average concentrations of the human plasma, with $c_{A}=4.25 \mathrm{~g} / \mathrm{dl}, c_{I}=1.25 \mathrm{~g} / \mathrm{dl}$ and $c_{F}=0.325 \mathrm{~g} / \mathrm{dl}$, at $X_{P}=100 \%$ plasma concentration in blood. When a protein is adsorbed on (released by) the surface, we keep its bulk concentrations constant by inserting (deleting) a protein of the same family in a randomly-chosen empty (occupied) space of the box. Every $60 \mathrm{~ns}$ we calculate the surface concentrations $C_{i}^{S}$ as the number of proteins of each family, adsorbed per unit surface, times their mass.

Experiments are usually carried out for highly diluted plasma, at concentration as small as $X_{P}=0.1 \%$, to slow down the adsorption rate to minutes or hours, allowing precise measurements. However, such low rates would decrease the statistics of our MD simulations. We, therefore, perform our simulations in conditions that are closer to those of practical interest, with $X_{P}$ as high as $100 \%, 50 \%$ and $25 \%$, by considering different sizes of the simulation box while keeping constant the initial number of proteins, their relative proportions, and the size of the adsorption surface. For each $X_{P}$ we average the results over fourteen independent runs, starting from independent initial configurations that have been equilibrated by applying pbc in any direction.

\section{Results and Discussion}

\subsection{Competitive Adsorption}

Result. In Figure 2 we show the surface concentrations of Alb, IgG, Fib and the total surfacae concentration as a function of time for total plasma concenttrations of $X_{P}=100 \%$ (Fig. 2a), $X_{P}=50 \%$ (Fig. 2b) and $X_{P}=25 \%$ (Fig. 2k). We find that protein surface concentrations $C_{i}^{S}$, for Alb and $\mathrm{IgG}$, are non-monotonic in time. In particular, for any considered $X_{P}, \mathrm{Alb}$ is the first protein that reaches the surface, due to its larger diffusive constant. This property induces an increase of $C_{A}^{S}$. When the second fastest and second most affine protein, $\mathrm{IgG}$, diffuses to the surface, it displaces $\mathrm{Alb}$, leading to a decrease of $C_{A}^{S}$ and an increase of $C_{I}^{S}$. Finally Fib, which is the slowest and most affine protein to the surface, takes over decreasing $C_{I}^{S}$ and increasing $C_{F}^{S}$. Each $C_{i}^{S}$ saturates toward an equilibrium value at long times, while the total surface concentration of proteins is saturated at early times.

Discussion: Differences with experiments. This behav-

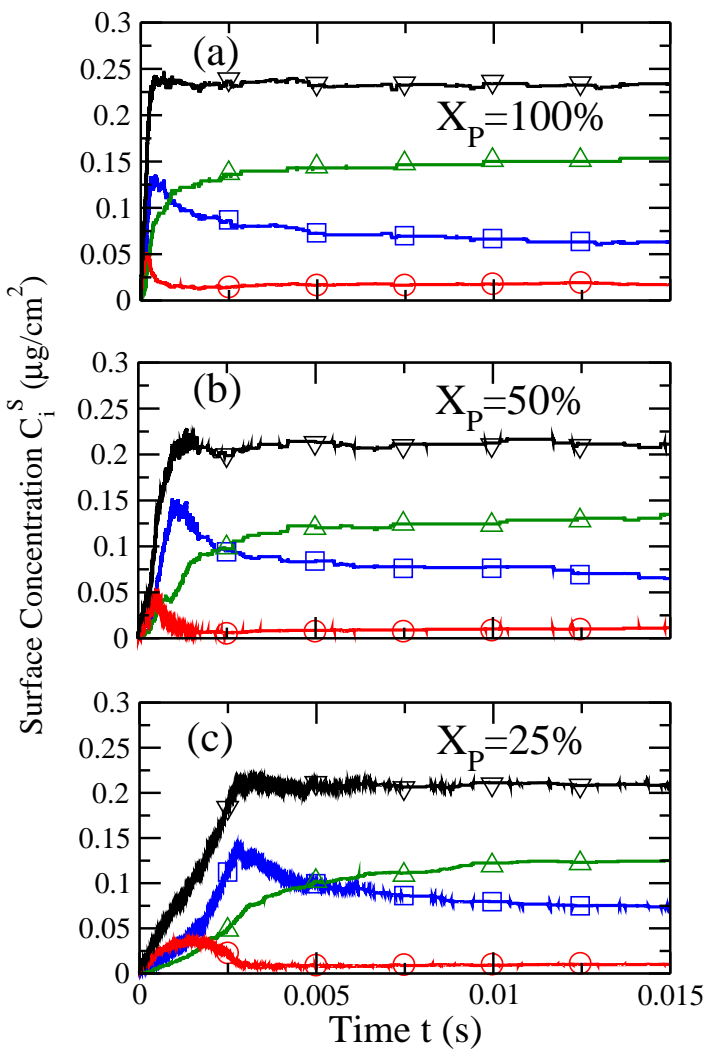

Fig. 2 Simulations at $T=300 \mathrm{~K}$ and (a) $X_{P}=100 \%$, (b) $X_{P}=50 \%$ and (c) $X_{P}=25 \%$ show that, at any considered dilution, surface concentration $C_{A}^{S}$ of $\mathrm{Alb}(\bigcirc), C_{I}^{S}$ of $\operatorname{IgG}(\square)$ and $C_{F}^{S}$ of Fib $(\Delta)$ are not all monotonic with time, while their $\operatorname{sum}(\nabla)$ is monotonic within our numerical precision. The concentrations are calculated every $60 \mathrm{~ns}$ (lines), while symbols are plotted only every $2.5 \mathrm{~ms}$ for sake of clarity. Bulk concentrations are as indicated in the text. Errors are smaller than symbol sizes.

ior qualitatively reproduces the Vroman effect, apart from the behavior of Fib that here is monotonic, while in experiments has a maximum due to the competitive adsorption with heavier and more surface-affine plasma proteins, like the high molecular weight kininogen, not included in our model ${ }^{11,41}$.

\subsection{Effect of Plasma Dilution}

Result. In Figure 3 we show the effect of plasma dilution on the surface concentrations by comparing each $C_{i}^{S}$ at different values of $X_{P}$. By increasing the dilution (i.e. reducing $X_{P}$ ) all the surface concentrations tends toward the same large-time limit (Fig. 3). However, when $X_{P}$ is reduced the dynamics of the process slows down. This is consistent with what is observed in experiments $\frac{42}{2}$ and was reproduced by kinetic models with displacement rates, "liberation" rates and semipermanently adsorbed state for Fib ${ }^{11}$. We can observe the slowing 


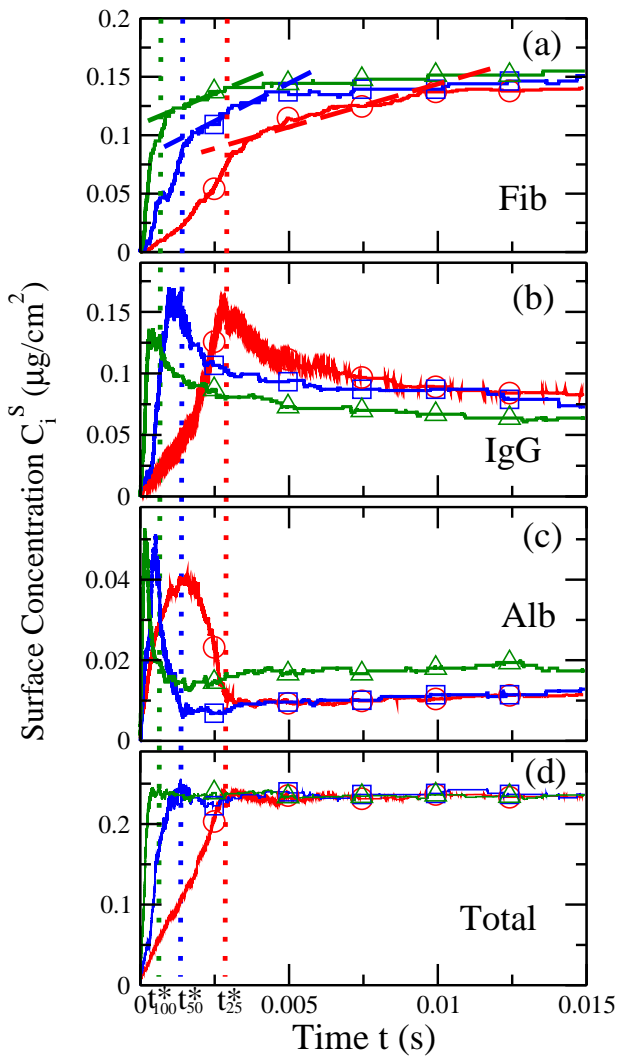

Fig. 3 Same data at $T=300 \mathrm{~K}$ as in in Fig. 2 now separated for (a) Fib, (b) IgG, (c) Alb, and (d) their sum at $X_{P}=100 \%(\Delta)$, $X_{P}=50 \%(\square)$, and $X_{P}=25 \%(\bigcirc)$. The time-scales increase for decreasing $X_{P}$. In all the panels the vertical dotted lines are guides for the eyes to mark characteristic times. In (a) the straight lines are a guide to the eyes for the linear regime of the three-steps kinetics of Fib adsorption. Note that the vertical scale of panel (c) is almost four times smaller than those in panels (a) and (b).

down not only for Fib (Fig. 3a), but also for the competing proteins IgG (Fig. 3b) and Alb (Fig. 35).

Discussion: Differences with Langmuir kinetics. It is interesting to observe that Alb concentration (Fig. 3k) reaches a shallow minimum at a time $t_{X_{P}}^{*}$ that depends on $X_{P}$ and approximately corresponds to that of maximum surface concentration of IgG (Fig. $3 \mathrm{~b}$ ). At these short-times $t<t_{X_{P}}^{*}$ the surface concentration of Fib increases more than linearly with time. This is more evident at low $X_{P}$ (Fig. 3 $\mathrm{a}$ ). This behavior has been predicted in other models for single protein adsorption including conformational changes and has been noted that it is not reproduced by standard Langmuir kinetics ${ }^{43}$. It can be understood as a consequence of the ability of Fib to adsorb in both its "laying down" and "standing up" conformation that is not captured by standard Langmuir kinetics.

\subsection{Three-steps adsorption of Fib}

Result. The stage at $t<t_{X_{P}}^{*}$ can be considered as the first step of Fib adsorption and occurs when the competitions with the other proteins is not strong, i.e. when the total surface concentration has not reached its saturation (Fig. 3 d d). The time $t_{X_{P}}^{*}$ coincides, within our numerical precision, also with the beginning of a "linear" regime, i.e. a regime of constant adsorption rate, for the Fib (Fig. 3a). This linear regime represents a second step in the Fib adsorption and precedes a third step during which $C_{F}^{S}$ saturates.

Discussion: Similarities with experiments and differences with Langmuir kinetics. This three-steps kinetics has been experimentally observed, and numerically reproduced, in single protein adsorptions on thin $\mathrm{SiO}_{2}$ layers, both hydrophilic or with an additional hydrophobic monolayer, at room temperature and at $37.5^{\circ} \mathrm{C}^{36.44}$. The authors of those works interpreted this behavior as a consequence of protein diffusion at the surface and of the occurrence of conformational changes. However, they did not study the case with competitive adsorption.

Here, instead, we observe that the regime of constant Fib adsorption rate coincides with the $\operatorname{IgG}$ desorption and the slow re-adsorption of Alb. This suggests that the reorganization of the proteins at the surface is likely to involve all the three families of proteins at the same time, in a way that is far more complicated than the usual two-states models based on kinetics equations with "transition" rate constants. Indeed, standard Langmuir kinetics would be able to predict the general trend of slowing down for increasing dilution ${ }^{45}$, but is unable to reproduce the three-steps kinetics, even in single protein adsorption, when conformational changes take place $36,43,44$.

Discussion: Interpretation in terms of competitive and "cooperative" adsorption. The second step starts, at $t_{X_{P}}^{*}$, when the total surface concentration is saturated (Fig. 3 $\mathrm{d}$ ). Therefore, new arriving Fib adsorbs in the "laying down" conformation if possible, or, with less energy gain but occupying less space, in the "standing up" conformation. Since at $t_{X_{P}}^{*}$ the $\mathrm{IgG}$ concentration is at its maximum, the probability that the new Fib adsorbs near a IgG (as in Fig 1d) is high, determining a strong repulsion between the two charged proteins. This repulsion is stronger than the attraction of IgG with the surface, determining the displacement of the $\mathrm{IgG}$ and the decrease of $C_{I}^{S}$.

This displacement leaves enough space on the surface for the adsorption of the smaller Alb that is abundant in suspension. As a consequence, $C_{A}^{S}$ increases. Despite Alb lower affinity to the surface, its small size allows the protein to fit onto the free surface without experiencing strong repulsion with the Fib. Therefore, at this stage Alb and Fib are not necessarily competing between them, but are both competing with the IgG. This stage can be described as "cooperative" adsorp- 


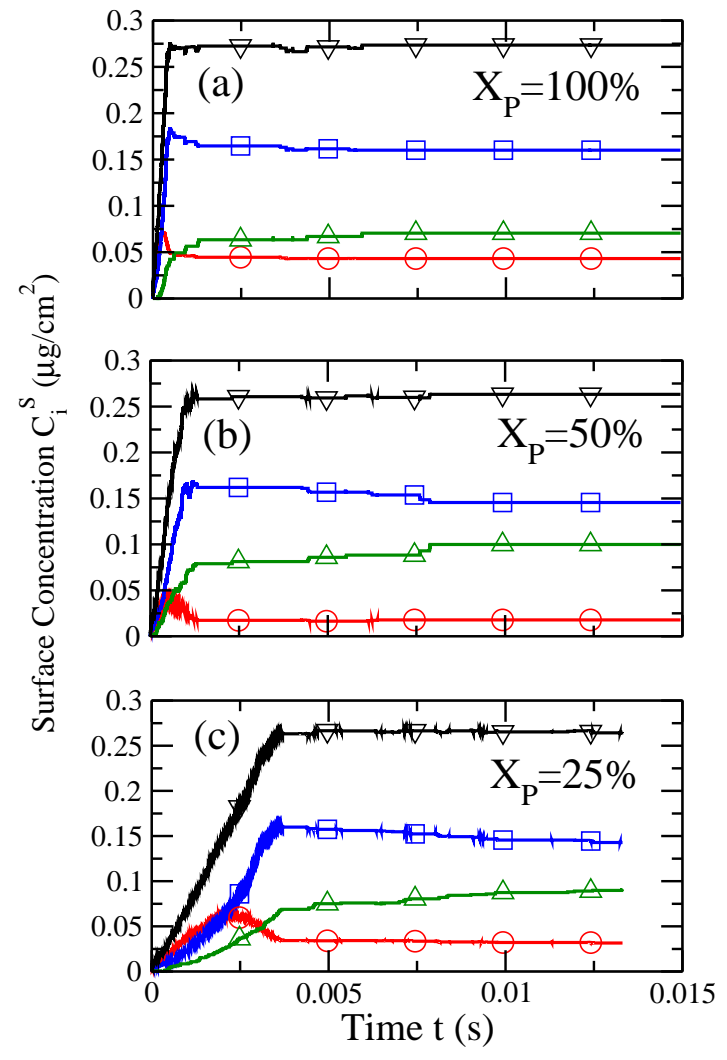

Fig. 4 Surface concentrations $C_{i}^{S}$ as function of time for $T=120 \mathrm{~K}$ at (a) $X_{P}=100 \%$, (b) $X_{P}=50 \%$ and (c) $X_{P}=25 \%$. Errors and symbols are as in Fig. 2] Alb $(\bigcirc), \operatorname{IgG}(\square)$, Fib $(\Delta)$ and their sum $(\nabla)$. At long time, $C_{I}^{S}>C_{F}^{S}$, with an inversion with respect to the standard conditions in Fig. 2 where $C_{F}^{S}>C_{I}^{S}$.

tion of Alb and Fib.

However, at larger time, when more Fib arrives to the surface, the competition is strong among all the three proteins. This induces the end of the re-adsorption of Alb and forces further conformational changes for the Fib (as in Fig 1). Our calculations support the identification of the third adsorption step, i.e. the end of the regime of constant Fib adsorption rate, with the end of the re-adsorption of Alb. This is more evident for the the lowest dilution, $X_{P}=100 \%$, while is more speculative for the other values of $X_{P}$.

\subsection{Effect of Energy Depletion}

Next, we study how energy depletion of the protein solution affects the sequence of adsorption. In experiments the energy is controlled by adding sodium azide, or other depletionenergy chemical agents, to the protein solution ${ }^{46}$. Here, for sake of simplicity, we decrease $T$, reducing the kinetic energy of the solution, but neglecting possible effects of the protein stability.

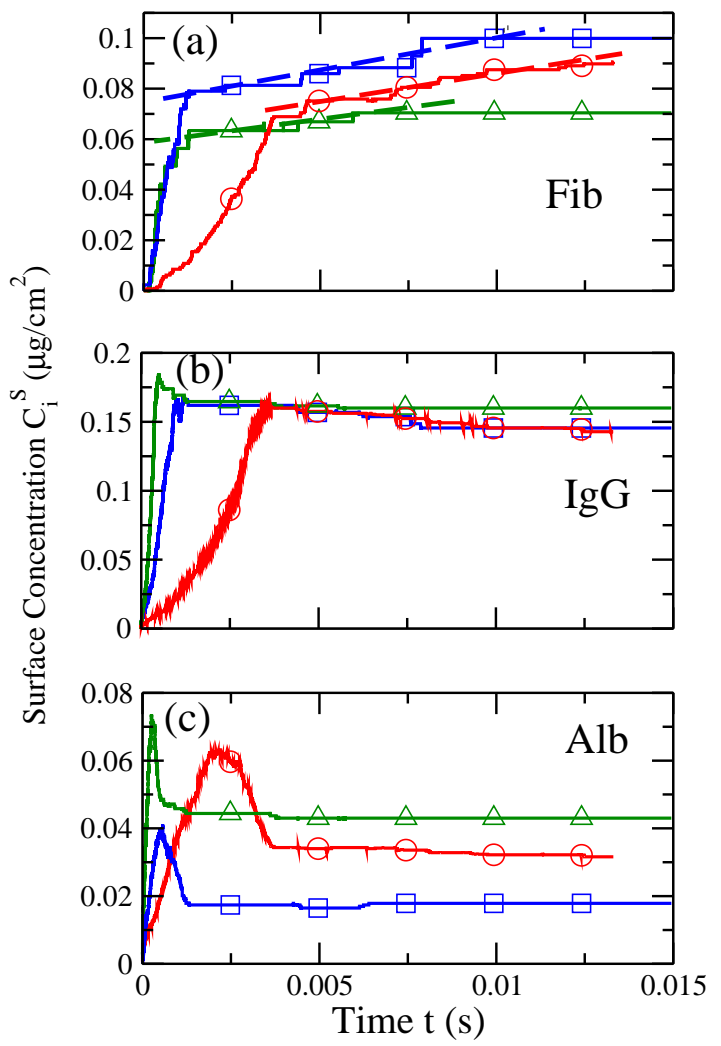

Fig. 5 Same data at $T=120 \mathrm{~K}$ as in Fig. 4 now separated for (a) Fib, (b) IgG and (c) Alb, at $X_{P}=100 \%(\Delta), X_{P}=50 \%$ ( $\square$ ), and $X_{P}=25 \%(\bigcirc)$. In (a) the straight dashed lines are guide to the eyes, showing that the linear regime is more extended than at $T=300 \mathrm{~K}$. For Alb the $C_{A}^{S}$ of saturation is non-monotonic with $X_{P}$.

Result: Inversion of the Vroman effect. In Figure 4 we show the surface concentrations as a function of time for temperature $T=120 \mathrm{~K}$ for different dilutions $X_{P}$. We find $(i)$ that, although the surface affinity of Fib is stronger than that for $\mathrm{IgG}$, the latter becomes the dominant protein adsorbed on the surface for long time scales; (ii) that, by changing $X_{P}$, the time scale of the process becomes longer, but the inversion of the protein concentration is always present. Hence, the energy depletion leads to an inversion of the Vroman effect.

Discussion: Effect on time-scales. By comparing the results at different energies, $k_{B} T$, and same $X_{P}$ (Fig. 2 and Fig. (4), we observe only a week energy-dependence of the times at which each $C_{i}^{S}$ reaches its maximum. Hence, the timescales of the process are mainly controlled by the total plasma concentration $X_{P}$, while the slowing-down due to the reduced diffusion seems to be less relevant.

Discussion: Effect on three-steps adsorption of Fib and Alb-Fib "cooperative" adsorption. We now compare the $C_{i}^{S}$ at different $X_{P}$ for the same protein, as shown in Figure 5 . We find that second step in the Fib adsorption is now more 
extended in time.

This result is consistent with what has been observed in the experiments from single protein absorption when conformational changes occur ${ }^{36}$. Moreover, our analysis for competitive adsorption shows, as for $T=300 \mathrm{~K}$, that the linear regime of Fib adsorption coincides with the end of the desorption of the Alb and the beginning of desorption of IgG. Therefore, we find also in this case evidences of "cooperative" behavior between Alb and Fib.

However, at $T=120 \mathrm{~K}$, the ability of Fib to displace IgG is much more limited than at $T=300 \mathrm{~K}$, because the displacement requires too much energy. This fact, on one hand, limits the adsorption of Fib, on the other hand does not allows Alb to re-adsorb. Nevertheless, the competition between Fib and $\mathrm{IgG}$ is enough to stop the desorption of Alb that now saturates at a value higher than for $T=300 \mathrm{~K}$ at any $X_{P}$.

Discussion: Effect on the long-time surface concentration. Another not intuitive result is that at $T=120 \mathrm{~K}$ the adsorption behavior is less regular than at $T=300 \mathrm{~K}$. For example, at the lowest $X_{P}=25 \%$ the Fib seems to adsorb more than at the highest, $X_{P}=100 \%$, and less than at the intermediate $X_{P}=50 \%$. A similar non-monotonic behavior characterizes also the Alb absorption, but now the $C_{A}^{S}$ is higher when $C_{F}^{S}$ is lower and vice versa.

These results suggest that, at long time, the strongest competition is between Alb and Fib, because IgG is almost not displaced from the surface. Furthermore, at $X_{P}=25 \%$ Fib does not reach the third step of adsorption, suggesting that the kinetics is so slow that it does not allow the Fib to perform large conformational changes.

\subsection{Change of the Bioenvironment}

Once we have understood that the protein layer covering the surface is controlled by the energy depletion of the system, it is interesting to ask if a sudden change of external conditions could induce a different composition of this layer, determining different biomimetics surface properties. This situation could occur, for example, when a medical device is manipulated in a bioenvironment whose composition is externally controlled during a surgery $\stackrel{47}{ }$. In particular, we study the case in which the system is first equilibrated under energy-depleted conditions and subsequently undergoes a sudden change that reestablishes the normal conditions.

Result. Figure 6 shows the effect of switching from an energy-depleted condition to a normal condition at time $t_{0}$. At short times, $t<t_{0}$, the energy-depleted system evolves until the equilibrium concentrations are reached. Under these conditions, as discussed in Section 4.4 (Fig. 4), the dominant protein is $\mathrm{IgG}$ instead of Fib.

At time $t_{0}$ we switch to normal conditions, forcing the system out of equilibrium. As a consequence, the system re-

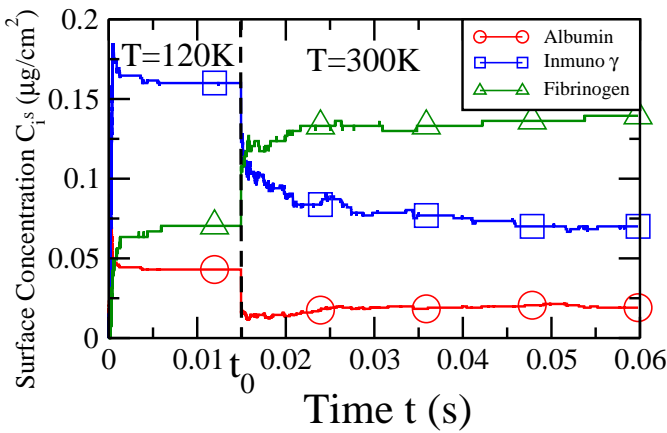

Fig. 6 The surface concentrations $C_{i}^{S}$, as a function of time for $X_{P}=100 \%$, is drastically affected when the system undergoes a sudden change from an energy-depleted condition to a normal condition. The vertical dashed line marks the time $t_{0}$ of the change. We control the energy of the solution by changing the external parameter $T$ from $T=120 \mathrm{~K}$ to $T=300 \mathrm{~K}$. Errors and symbols are as in Fig. 2

enters a transitory situation in which the concentrations $C_{i}^{S}$ evolve until they reach their new equilibrium values at long times. In the specific case considered here, we observe a fast change in the surface concentrations, with $C_{F}^{S}$ of Fib overcoming $C_{I}^{S}$ of $\mathrm{IgG}$, being the first, under normal conditions, more stable on the surface than the second. The final equilibrium concentrations are reached at large times, $t>t_{0}$.

Discussion. Together with the changes of $C_{F}^{S}$ and $C_{I}^{S}$, we observe also a sudden change in $C_{A}^{S}$ of $\mathrm{Alb}$, between the two equilibrium concentrations characteristics of the two values of the external parameters $T$. However, $C_{A}^{S}$ always equilibrates to a value that is smaller then $C_{I}^{S}$ and $C_{F}^{S}$, consistent with its long-time values in Fig. 2] and Fig. 4

By decreasing $X_{P}$, we find the same qualitative behavior for a sudden energy-change, but with the transient regime extending to longer times, consistent with Fig. 4. Hence, at experimental values of $X_{P}$ the switching behavior would occur on time scales that are comparable to those characteristic of the Vroman effect.

\subsection{Reversible vs Irreversible Adsorption}

We remark that our predictions about inverting the Vroman effect by changing the experimental control parameters should hold only if the protein adsorption on the surface is reversible. If the adsorption is, instead, irreversible the change of external parameters should not lead to a new composition of the protein layer. Indeed, under many practical conditions of interest for blood plasma, it would appear that the binding is indeed mostly irreversible 2,48 . Hence, the switching protocol proposed above represents a possible experimental way to evaluate how strongly irreversible is the adsorption process on a specific surface. 
For an irreversible adsorption process, our findings predict that by appropriately controlling the parameters of the protein solution, such as the amount of depleted energy, it is possible to engineering a specific biomimetic covering of a surface. Due to the irreversibility, the proteins, once adsorbed, cannot easily desorb from the surface, even if the external conditions are modified. Therefore, it is feasible to cover a device surface with any desired protein composition, targeted to a specific biomimetic property, by selecting an appropriate initial condition. Subsequently, the device could be used under physiological conditions with no further changes of the protein cover and its biomimetic properties.

\section{Conclusions}

We study, by MD simulations of a coarse-grained model, the Vroman effect for a ternary protein solution mixture, with Alb, IgG and Fib, in contact with a hydrophobic surface. We show that the effect is the consequence of the differences among the proteins properties: mass and size, affinity, diffusion constant, conformational changes. These differences lead to a process of competitive adsorption on a surface, in which the different families of proteins occupy sequentially the surface, replace each other and diffuse at the surface, until an equilibrium situation is reached. By decreasing the total concentration of protein in the solution, keeping the relative concentrations fixed, the time scales of the process increase and the maxima of surface concentration for each family of proteins occur at longer times.

Our model confirms the intuitive understanding that the sequence of surface occupation is a consequence of a competition between the smaller and faster, but less affine, proteins with the more affine, but bigger and slower, proteins. For example, we test that by increasing the Alb affinity, or artificially setting to the same value all the diffusion constants, the Vroman effect disappears. Therefore, affinity and diffusion constant are relevant protein parameters for the effect as can be deduced by standard kinetics equation models. Nevertheless, our model reveals that the mechanisms of competitions are likely to be more complex that what the intuition would suggest, when conformational changes occurs, with regimes during which small and large proteins are not necessarily competing between them, but are both competing with others in solution in a "cooperative" way. This feature appears here related to a characteristic three-steps adsorption of Fib that presents similarities with experiments and differences with Langmuir kinetics.

We find that the protein surface concentrations at equilibrium depend on external control parameters. In particular, we find that energy depletion induces a drastic change in the composition of the covering protein-layer, leading to an inversion of the Vroman effect. Our results show that the inversion can be used to quantify how strongly irreversible is the process of surface adsorption of the proteins, an information useful in studies of thromboembolic events ${ }^{49}$. Furthermore, these results suggest the possibility of engineering the composition of the protein layer covering a surface in a controlled way, a feature particular relevant in biomimetic applications.

\section{Acknowledgments}

We thank C. Aberg, F. Baldelli Bombelli, M. P. Monopoli and O. Vilanova for discussions. We acknowledge the support of EU FP7 grant NMP4-SL-2011-266737; PV and GF of Spanish MEC grant FIS2012-31025.

\section{References}

1 T. Cedervall, I. Lynch, S. Lindman, T. Berggård, E. Thulin, H. Nilsson, K. A. Dawson and S. Linse, Proceedings of the National Academy of Sciences, 2007, 104, 2050-2055.

2 M. P. Monopoli, D. Walczyk, A. Campbell, G. Elia, I. Lynch, F. Baldelli Bombelli and K. A. Dawson, Journal of the American Chemical Society, 2011, 133, 2525-2534.

3 J. A. Kim, C. Aberg, A. Salvati and K. A. Dawson, Nat Nano, 2012, 7, 62-68.

4 D. Walczyk, F. B. Bombelli, M. P. Monopoli, I. Lynch and K. A. Dawson, Journal of the American Chemical Society, 2010, 132, 5761-5768.

5 G. Oberdörster, A. Elder and A. Rinderknecht, J. Nanosci. Nanotechnol., 2009, 9, 4996-5007.

6 P. Tengvall, I. Lundström and B. Liedberg, Biomaterials, 1998, 19, $407-$ 422.

7 L. Vroman, Nature, 1962, 196, 476-477

8 J. L. Ortega-Vinuesa and R. Hidalgo-Álvarez, Biotechnology and Bioengineering, 1995, 47, 633-639.

9 M. Holmberg and X. Hou, Langmuir, 2009, 26, 938-942.

10 D. R. Jackson, S. Omanovic and S. G. Roscoe, Langmuir, 2000, 16, 5449_ 5457.

11 C. A. LeDuc, L. Vroman and E. F. Leonard, Industrial \& Engineering Chemistry Research, 1995, 34, 3488-3495.

12 R. Green, M. Davies, C. Roberts and S. Tendler, Biomaterials, 1999, 20, 385-391.

13 H. Noh and E. A. Vogler, Biomaterials, 2007, 28, 405-422.

14 Y.-X. Ding and V. Hlady, Croat Chem Acta., 2011, 84, 193-202.

15 B. Lassen and M. Malmsten, Journal of Colloid and Interface Science, 1996, 180, 339-349.

16 B. Lassen and M. Malmsten, Journal of Colloid and Interface Science, 1997, 186, 9-16.

17 N. Barnthip, P. Parhi, A. Golas and E. A. Vogler, Biomaterials, 2009, 30, 6495-6513.

18 C. F. Lu, A. Nadarajah and K. K. Chittur, Journal of Colloid and Interface Science, 1994, 168, 152-161.

19 J. L. Brash and T. A. Horbett, in Proteins at Interfaces, American Chemical Society, Washington, DC, 1995, pp. 1-23.

20 S. M. Slack and T. A. Horbett, in The Vroman Effect, American Chemical Society, Washington, DC, 1995, pp. 112-128.

21 P. Dejardin, P. ten Hove, X. J. Yu and J. L. Brash, Langmuir, 1995, 11, 4001-4007.

22 S.-Y. Jung, S.-M. Lim, F. Albertorio, G. Kim, M. C. Gurau, R. D. Yang, M. A. Holden and P. S. Cremer, Journal of the American Chemical Society, 2003, 125, 12782-12786. 
23 S. L. Hirsh, D. R. McKenzie, N. J. Nosworthy, J. A. Denman, O. U. Sezerman and M. M. M. Bilek, Colloids and Surfaces B: Biointerfaces, 2013, 103, 395-404.

24 P. A. Cuypers, G. M. Willems, H. C. Hemker and W. T. Hermens, Annals of the New York Academy of Sciences, 1987, 516, 244-252.

25 I. Lundström and H. Elwing, Journal of Colloid and Interface Science, 1990, 136, 68-84.

26 P. Roach, D. Farrar and C. C. Perry, Journal of the American Chemical Society, 2005, 127, 8168-8173.

27 T. Wei, M. A. Carignano and I. Szleifer, The Journal of Physical Chemistry B, 2012, 116, 10189-10194.

28 V. Tozzini, Current Opinion in Structural Biology, 2005, 15, 144-150.

29 M. D. Bale, D. F. Mosher, L. Wolfarht and R. C. Sutton, Journal of Colloid and Interface Science, 1988, 125, 516-525.

30 G. Franzese, V. Bianco and S. Iskrov, Food Biophysics, 2011, 6, 186-198.

31 P. Gallo, D. Corradini and M. Rovere, Physical Chemistry Chemical Physics, 2011, 13, 19814-19822.

32 M. Lund, P. Jungwirth and C. E. Woodward, Physical Review Letters, 2008, 100, 258105-.

33 S. Ravichandran and J. Talbot, Biophysical journal, 2000, 78, 110-120.

34 M. R. Oberholzer, N. J. Wagner and A. M. Lenhoff, The Journal of Chemical Physics, 1997, 107, 9157-9167.

35 C. Ruggiero, M. Mantelli, A. Curtis, S. Zhang and P. Rolfe, 1999, 37, 119-124.
36 M. Bellion, L. Santen, H. Mantz, H. Hähl, A. Quinn, A. Nagel, C. Gilow, C. Weitenberg, Y. Schmitt and K. Jacobs, Journal of Physics: Condensed Matter, 2008, 20, 404226.

37 M. Malmsten, D. Muller and B. Lassen, Journal of Colloid and Interface Science, 1997, 193, 88-95.

38 S.-K. Ma, in Statistical Mechanics, World Scientific, 1985, ch. 9.

39 F. Galisteo-González, A. Martín-Rodríguez and R. Hidalgo-Alvarez, Colloid \& Polymer Science, 1994-03-01, 272, 352-358.

40 C. Mücksch and H. M. Urbassek, Langmuir, 2011, 27, 12938-12943.

41 P. Turbill, T. Beugeling and A. Poot, Biomaterials, 1996, 17, 1279-1287.

42 J. L. Brash and P. ten Hove, Thrombosis and Haemostasis, 1984, 51, 326.

43 Z. Adamczyk and P.-1. Weronski, Langmuir, 1995, 11, 4400-4410.

44 A. Quinn, H. Mantz, K. Jacobs, M. Bellion and L. Santen, EPL, 2008, 81, year.

45 R. I. Masel, in Principles of adsorption and reaction on solid surfaces, Wiley Interscience, 1996, p. 240.

46 K. Shapero, F. Fenaroli, I. Lynch, D. C. Cottell, A. Salvati and K. A. Dawson, Mol. BioSyst., 2011, 7, 371-378.

47 A. N. Cloud, S. Kumar, M. Kavdia, H. H. Abu-Safe and M. H. Gordon, Surface and Coatings Technology, 2008, 203, 913-917.

48 M. Lundqvist, J. Stigler, T. Cedervall, T. Berggård, M. B. Flanagan, I. Lynch, G. Elia and K. Dawson, ACS Nano, 2011, 5, 7503-7509.

49 S. Uniyal and J. L. Brash, Thrombosis and Haemostasis, 1982, 47, 28590 